\title{
Factores asociados a la construcción de marca del artista musical en Bogotá con aplicativos móviles
}

\section{Factors tied to the Brand Construction of the Music Artist in Bogotá with Mobile Applications}

\author{
Hernán David Arias Cortés \\ Profesional en Medios Audiovisuales por la \\ Institución Universitaria Politécnico Grancolombiano \\ y Magíster en Mercadeo Estratégico por la \\ Institución Universitaria Politécnico Grancolombiano. \\ Docente en la Institución Universitaria Politécnico \\ Grancolombiano y la Universidad Jorge Tadeo Lozano. \\ Correo electrónico: davargot001@yahoo.com
}

\author{
Gary Hernández Guerrero \\ Publicista y productor radial por la Universidad \\ Jorge Tadeo Lozano de Bogotá, especialista en \\ Gerencia de Publicidad y magister en Mercadeo \\ Estratégico por la Institución Universitaria \\ Politécnico Grancolombiano. Actual coordinador \\ del equipo creativo del Canal RCN Novelas. \\ Correo electrónico: alcance-publicitario@hotmail.com
}

\begin{abstract}
Leonardo Ortegón Cortázar
Magíster en Psicología del Consumidor y doctorando en Marketing. Docente de la Facultad de Mercadeo, Comunicación y Artes de la Institución Universitaria Politécnico Grancolombiano.

Correo electrónico: lortegon@poligran.edu.coMáster en Marketing Instituto Superior Politécnico Gaya, Instituto Superior de Contabilidade e Administração do Porto

Correo electrónico: jdsantos@iscap.ipp.pt
\end{abstract}

\begin{tabular}{|c|c|c|}
\hline $\begin{array}{l}\text { FECHA DE RECEPCIÓN: } \\
\text { FECHA DE REVISIÓN: } \\
\text { APROBACIÓN: }\end{array}$ & $\begin{array}{l}28 \text { DE ENERO DE } 2014 \\
3 \text { DE FEBRERO DE } 2014 \\
15 \text { DE JUNIO DE } 2014\end{array}$ & $\begin{array}{l}\text { Para CitAR ESte artículo / to CITE thIS ARTICle } \\
\text { Arias Cortés, H., Hernández Guerrero, G. y Ortegón } \\
\text { Cortázar, L. (2014). Factores asociados a la cons- } \\
\text { trucción de marca del artista musical en Bogotá con } \\
\text { aplicativos móviles. Poliantea, } 10 \text { (19), pp. 61-91. }\end{array}$ \\
\hline
\end{tabular}




\section{Resumen}

El desarrollo tecnológico ha generado importantes cambios en cuanto a consumo, promoción y comercialización de artistas musicales. Este artículo buscó identificar los aspectos más significativos de la creación de marca por parte del artista musical, explorando su relación con el marketing digital para potencializar el branding y las motivaciones que estimulan el consumo de productos musicales en un segmento conocido como los millenials (milénicos). Los resultados dan evidencia de estrategias comunicativas y comerciales que están implementando artistas, productores y comunicadores relacionados con la industria musical para lograr la construcción de una marca musical por medios digitales.

Palabras clave: industria musical, mercadeo digital, aplicativos móviles, construcción de marca, branding digital.

\section{Abstract}

Technological development has generated important changes regarding the consumption, promotion, and commercialization of music artists. This article aims to identify the most meaningful aspects of brand creation from the music artist by exploring his/her relationship with digital marketing to reinforce branding and the motivation that stimulates the consumption of musical products in a segment known as millennials. The results show communicative and commercial strategies that artists, producers, and communicators are implementing and that are related to the music industry in order to build a music brand through digital media.

Keywords: Music industry, digital marketing, mobile applications, brand construction, digital branding. 


\title{
Factores asociados a la construcción de marca del artista musical en Bogotá con aplicativos móviles
}

\section{Factors tied to the Brand Construction of the Music Artist in Bogotá with Mobile Applications}

\author{
Hernán David Arias Cortés \\ Institución Universitaria Politécnico Grancolombiano \\ Gary Hernández Guerrero \\ RCN Novelas \\ Leonardo Ortegón Cortázar \\ Institución Universitaria Politécnico Grancolombiano
}

\section{Introducción}

Muchas de las expresiones sonoras dentro del mercado actual de la música local son el resultado de una mezcla entre ritmos nativos y la constante influencia de músicas foráneas. Sonidos provenientes de distintas latitudes, como la música electrónica, el rap, el pop, el rock, la salsa y el jazz, entre otros, han sido fusionados con ritmos muy tradicionales de los folclores, y han adoptado incluso estilos extranjeros como parte de la cultura musical popular de un país. Esta fusión es también una respuesta a las tendencias que promueve la globalización musical (Mukuma, 2010), tras lo cual genera un desarrollo comercial, tecnológico, social, político e ideológico (Reyes, 2001).

Por otro lado, desde comienzos de siglo XXI, los avances en la tecnología han facilitado la promoción y comercialización de bienes y servicios, así como 
la disminución de costos de inversión publicitaria en los medios tradicionales por parte de las marcas, las cuales han enfocado sus esfuerzos de marketing hacia los medios digitales, con lo cual ha respondido a la aparición de nuevas tendencias de consumo en la industria musical. Esta investigación se centra en los factores que determinan la construcción de marca en los artistas musicales con las tecnologías de la información.

\section{Marco teórico}

\section{El marketing y la música}

El marketing moderno se considera como un proceso social y administrativo mediante el cual los individuos y los grupos obtienen lo que necesitan y desean (Kotler et al., 2007). La American Marketing Association (2013) articula el marketing como la actividad, un conjunto de instituciones y procesos para crear, comunicar, entregar e intercambiar ofertas que tienen valor para los consumidores, los socios y la sociedad en general.

Como complemento de la anterior definición, entendemos el marketing como aquella profesión que, al valerse de elementos de la administración, economía, contabilidad, estadística, publicidad e investigación
(Hoyos, 2008), es capaz de identificar los intereses y las necesidades de un cliente, usuario o prosumidor, comprendido este último como parte de aquellas audiencias participativas que se "vuelven usuarias, productoras y emisoras" (Mantecón, 2010), "de sus propios productos, sin necesidad de intermediarios" (Carrero y Pulido, 2012).

$\mathrm{Al}$ aplicar el marketing a la industria musical para el fortalecimiento y la proyección del artista o del producto musical, se expande una serie de posibilidades que abarcan el uso de las reconocidas 4P, consideradas por McCarthy desde 1960 (Sileverman, 1995) e introducidas por Neil Borden en 1953 bajo el término de marketing mix (Van Waterschoot y Van den Bulte, 1992). Estas tácticas de marketing (promoción, precio, plaza y producto) han sido funcionales hasta el presente, y son parte del tradicional modelo de negocio en la industria musical (Vaccaro y Cohn, 2004). Sin embargo, se ha considerado la necesidad de categorizar con detalle el tradicional concepto de las $4 \mathrm{P}$, complementándolo con un modelo de $8 \mathrm{P}$ que incluye personal, activos fijos (physical assets), procesos y personalización (Goldsmith, 1999). 
El modelo adoptado por la industria de la música para la emisión, producción y comercialización musical se logró durante el último siglo, principalmente con invenciones como el gramófono, el fonógrafo, la radio, la TV, los LP, las cintas de casetes, los videoclips, el CD, el DVD y el Blueray, sin olvidar los conciertos en vivo, fundamentales a lo largo de la historia para la promoción de las producciones y artistas musicales. Estos fueron la base que sustentaba a la industria musical desde finales del siglo XX hasta comienzos del XXI (Ogden, Ogden y Long, 2011). Como consecuencia de los desarrollos tecnológicos relacionados con internet 3.0 (Carrero y Pulido, 2012), dicho modelo ha cambiado. Las recientes preferencias en el consumo musical con espectáculo en vivo y la promoción en línea: live social media, music $g a$ ming, e-ticketing, e-learning, bigdata musical, streaming multiplataforma, crowdfunding, aplicaciones y generación de contenidos multiformatos (Cereijo, 2014), son en la actualidad los mayores motores que impulsan un moderno modelo de negocio en la industria musical, por medio de la venta digital de canciones en plataformas como iTunes, descarga libre de archivos de forma legal en portales como
ReverbNation, reproducción gratuita de archivos sonoros en plataformas como SoundCloud, servicios de música por demanda en tiempo real (streaming) por medio de servicios como Pandora, Rhapsody, Spotify y Deezer, aplicativos de reconocimiento musical como Shazam y canales de interacción social para compartir videos y sonidos como Facebook, Myspace y YouTube, por nombrar los más populares (http://www.aliadodigital. com/2010/05/consumo-musical-youtube-es-mas-importante-que-myspace/). Otros dispositivos, como los computadores portátiles, las tabletas, los teléfonos inteligentes (smartphones) y las consolas de videojuego con sus respectivas formas de uso, son las herramientas que más utiliza el público para el consumo de productos musicales actualmente, por lo cual marcan una tendencia creciente en los ámbitos global (Cereijo, 2014) y local que varía según los aspectos demográficos de cada región (Colombia, Ministerio de las Tecnologías y la Información, 2010).

Es importante balancear los aspectos mercadotécnicos y los creativos en el escenario musical. Flores, Pérez y Blanco (2010) exponen la transformación que ha tenido la teoría del marketing de la cultura durante 
los últimos cuarenta años, incluso la música como parte de las expresiones culturales de una población. Destacan la influencia romántica sobre la cultura, caracterizada por la celebración de la libre expresión de la imaginación y el énfasis en la autenticidad de las emociones, aplicada fuertemente desde principios de la década de 1970 en las expresiones artísticas, que ubica al artista como el mayor decisor de las características de su producto, sin que importaran necesariamente las preferencias o necesidades de su público. Entre los defensores de este argumento donde el producto artístico debe siempre permanecer por encima de los intereses del mercado, se encuentra la autora Elizabeth Hirschman (1983), quien argumenta que el artista debe concebir su producto conforme a sus emociones y pensamientos lo demanden, independientemente de la aceptación que el público pueda tener sobre este, respetando la libertad para aceptarlo o rechazarlo. Hirschman también propone presentar al artista inicialmente como consumidor de su propia obra, sosteniendo que si el producto responde a las necesidades cognitivas y emocionales de su cliente - en este caso el artista-, el producto podrá hacerse disponible para el resto de los consumidores con el marketing mix (Van Waterschoot y Van den Bulte, 1992).

Contrastando esta posición sobre la forma en que el marketing debe abordar la actividad cultural, Flores, Pérez y Blanco (2010) citan el trabajo de Solomon, Polegato y Zaichkowsky (2009), quien introdujo una conceptualización del sistema de producción cultural, en la que incorpora tres subsistemas: el creativo, el de gestión y el de comunicación. Sobre estos participa un grupo de individuos y organizaciones que crean y comercializan productos culturales. En este orden de ideas, el concepto de marketing se aplica de forma completa, en especial cuando le es otorgada la decisión de crear un producto cultural al propio mercado. En contraste, Boorsma (2006) reconoce al consumidor como una pieza fundamental que complementa el producto final artístico, considerando el arte como un espacio relacional entre el productor inicial (artista) y el coproductor final (público), con lo cual se potencia la interacción social entre las partes.

Ogden, Ogden y Long (2011) exponen que en evidencias como los jeroglíficos se ha comprobado la presencia de la música en la humanidad desde hace 36000 millones de años 
aproximadamente. Además exponen que en $313 \mathrm{~d}$. C. se incrementó la comercialización musical ligada al auge del cristianismo en Europa; estilos musicales como el canto llano o canto gregoriano fueron de fácil repetición entre los integrantes de la comunidad eclesiástica con una notación musical aplicada en ese entonces que permitía tener un control sobre la comunidad religiosa alrededor del mundo. Más adelante, con el surgimiento del mecenazgo durante el siglo XV financiado por las clases altas de la sociedad, se permitía el crecimiento profesional del músico dándole acceso a ciertos beneficios para mejorar su calidad de vida.

La carrera del artista musical se empezó a formar con la remuneración económica de las producciones y financiación en actividades especiales, tras lo cual se consiguió un vínculo muy fuerte entre el músico y el público. Posteriormente a ello, gracias a la creación de herramientas de captura sonora, como el fonógrafo y el gramófono, a finales del siglo XIX se logró que la audiencia tuviese una nueva forma de entretenimiento.

El aporte de Guillermo Marconi, con el desarrollo de la telegrafía sin hilos, dio origen a las bases de la radiodifusión. Posteriormente,
Reginald Aubrey Fessenden consiguió realizar la primera transmisión de sonido con antenas de radiotelegrafía, con lo cual se dio inicio a una nueva era en las comunicaciones. Con la capacidad para cruzar fronteras por medio de las ondas electromagnéticas, la música tuvo un nuevo medio para promocionarse de forma masiva junto con sus intérpretes (López, 2005).

\section{Construcción de marcas musicales}

La marca y la forma en que incide en los usuarios ha sido motivo de estudio a lo largo de los años por diferentes autores. Aaker y Joachimsthaler (2005) consideran la marca como activo cuando se trata de forma estratégica o a largo plazo y como imagen y de forma táctica o a corto plazo (postura financiera y sensitiva). En este trabajo, se ha contemplado abordar la marca como el conjunto de elementos integrados en una estructura de imagen y significado que afectan, por medio de algún sentido, a quien se dirige y que establecen la identidad de quien la posee (visual, sonora, tangible, olfativa o gustativamente), a diferencia de la concepción de marca como activo o valor financiero. Se consideran los 
estímulos sensoriales planificados minuciosamente como provocadores de las preferencias en los consumidores, diferenciando la marca de su competencia (Gobé, 2005).

\section{El marketing digital y la generación milénica}

El constante uso de recursos tecnológicos y medios móviles digitales para el desarrollo de la comunicación ha generado un cambio en las tácticas de marketing utilizadas tradicionalmente por las marcas, las cuales buscan acercarse a sus usuarios con los nuevos dispositivos. En consecuencia, un alto porcentaje de estos que utilizan las nuevas tecnologías se encuentran dentro de la denominada generación $\mathrm{Y}$ o milénica, nacidos entre 1980 y 1995 (Ng, Schweitzer y Lyons, 2010). Dentro de los hábitos de este segmento de la población se encuentran la preferencia por el uso de plataformas como internet, el menor uso de medios tradicionales como la radio y la TV, interactuar con otros usuarios por medio de comentarios, influenciar o ser influenciados con canales de interacción social, ocupar una gran cantidad del tiempo en línea, tener preferencia por la comunicación vía correo electrónico o mensajería instantánea y evitar los mensajes publicitarios del tipo ventanas emergentes en la web (Taken, 2012).

Por otro lado, son muchos los beneficios que han llevado al marketing digital a ser parte esencial en las campañas de mercadeo estratégico, con lo cual se ha permitido de forma precisa el cumplimiento de metas claras y específicas que pueden calcularse por medio de métricas que posibilitan medir resultados de forma detallada (Libreros, 2013). La evolución de las comunicaciones y la aprehensión de las tecnologías de la información y comunicación (TIC) por parte de la población mundial han permitido tener un mercado activo constantemente interesado en consumir información de forma inmediata. Identificando las necesidades del mercado real y potencial (Barrere, 2012), las empresas pueden segmentar y orientar mucho mejor sus campañas de comunicación llegando de forma directa al público objetivo con una inversión más efectiva que la realizada en medios tradicionales.

Los mensajes publicitarios en internet iniciaron en forma de anuncios hacia 1994 y se publicitaba con estrategias denominadas pay per click. Los anuncios en teléfonos móviles fueron 
implementados en 1997. Para 2000 la inversión publicitaria relacionada con el tema digital había alcanzado niveles superiores con respecto a otros medios (http://hipertextual.com/archivo/2011/12/historia-publicidad/). La creación de portales web se destacaron en aquella época al igual que los dominios puntogot, puntocom y un servicio ofrecido por Google denominado AdWords, motor de búsqueda efectivo con respecto a la información que requiriera el usuario, que permite el relacionamiento de ciertas palabras con empresas o productos que buscan tener relevancia en la web.

Con la llegada de YouTube inicia la interactividad de los videos en directo, con lo cual se genera mayor participación por parte del consumidor. Las marcas encontraron en este portal una forma de llegar al mercado de forma más directa y personalizada (Ogden, Ogden y Long, 2011).

Tanto la música como los videos en directo han sido herramientas importantes en la decisión de compra en línea de productos, lo que ha servido, en muchas ocasiones, para generar diferentes emociones que influyen en la percepción de los consumidores respecto de algún producto. Sin embargo, uno de los aspectos que más incide en la compra en directo es la confiabilidad que brinde el portal web, ya sea por el diseño, ya sea por la identidad gráfica utilizada como elementos que podrían mejorar la probabilidad de compra (Kotler, 1973).

El ritmo y el tono de la música pueden estimular y aumentar el tiempo de compra del usuario que busca adquirir productos o servicios con los medios digitales (Ding y Lin, 2012). Estos mismos autores sugieren que mayor es el efecto cuando se utiliza sobre productos hedónicos en comparación con los utilitarios. Los productos hedónicos aluden a la diversión y el juego, mientras que los productos utilitarios no estimulan un sentimiento que incentive al consumidor a adquirirlo (Ding y Lin, 2012). De esta manera, con el desarrollo de los medios digitales, la música y sus estrategias de promoción se han tenido que transformar, cambiando a su vez el comportamiento de los consumidores, los cuales modifican sus preferencias según la experiencia con el producto (Schiffman y Kanuk, 2010).

Un ejemplo es lo que sucedió desde 1999 con el nacimiento de Napster (Ogden, Ogden y Long, 2011), portal que revolucionó la industria discográfica en el mundo, puesto que entregó al mercado la facilidad para descargar gratuitamente cualquier 
tipo de música. Esto llevó a muchos sellos disqueros a la quiebra. En 2001 la industria musical tuvo que cambiar las tácticas de marketing que utilizaba para promover a las bandas musicales y sus respectivas producciones. Dispositivos digitales como el iPod y portales web para la compra de música como iTunes Store contribuyeron a este cambio. En abril de 2003, con el lanzamiento del servicio iTunes, se calculó un promedio de 14 millones de canciones descargadas en los primeros seis meses, lo que evidencia de un cambio significativo en la forma de adquirir música (Vaccaro y Cohn, 2004). Para finales de 2006, las ventas de iPods ascendieron a 21 millones de unidades, $50 \%$ más que las ventas del año anterior (Brookshire, 2007). Una situación que ha surgido a partir del cambio en el consumo de música es la preferencia de los usuarios por la portabilidad que ofrecen los dispositivos móviles personales (Shankar y Balasubramanian, 2009). En este sentido, se percibe un fortalecimiento por parte del artista de diferenciarse en el mercado y que le permita llegar a la audiencia de forma directa, manteniendo una comunicación constante con el público.

Actualmente, se busca conseguir un posicionamiento en el mercado para ofrecer una propuesta de valor que les permita a los artistas musicales ser competitivos, con lo cual logran diferenciarse en el mercado no solo supliendo las necesidades de los consumidores, sino también conociendo las opiniones y manteniendo mayor contacto con los seguidores.

Otro de los propósitos del presente estudio es integrar dos perspectivas: la de los profesionales relacionados con el ámbito musical (productores de radio, músicos y gerentes de marketing digital) y la de un segmento de público joven, el cual es el que más utiliza las nuevas tendencias de información digital (Rodríguez, 2012).

De acuerdo con Smith (2011), la reflexión teórica sobre el mercadeo de la música se ha enfocado en las tácticas utilizadas para promoción de una obra o artista, evidenciadas dentro del mix de mercadeo, por lo cual ha dejado de lado el estudio de los factores de construcción de marca con los nuevos medios de interacción y comunicación. Esto conduce a un mercadeo basado en el relacionamiento y apoyado por los aplicativos, tras lo cual lo optimiza para lograr generar vínculos más fuertes y productivos.

De acuerdo con los anteriores argumentos, se percibe la necesidad de 
aportar información respecto a cuáles son los factores asociados a la construcción de marca del artista musical con aplicativos móviles.

Así, el anterior planteamiento de investigación busca cumplir los siguientes objetivos:

- Explorar los factores asociados a la construcción de marca del artista musical.

- Descubrir los factores que favorecen la construcción de marca del artista musical en dispositivos móviles digitales.

- Detectar el nivel de conocimiento del artista en cuanto al uso de dispositivos móviles para la construcción de una marca musical.

- Identificar las características que motivan la preferencia de un artista musical.

- Explorar los hábitos de consumo de producciones musicales con aplicativos móviles por parte del público investigado.

Para proporcionar información válida y confiable que resuelva los propósitos de este estudio, se propuso una investigación de carácter exploratorio descriptivo, que combina dos técnicas de investigación.

\section{Método}

La investigación realizada corresponde a un diseño exploratorio descriptivo con uso de las técnicas de entrevistas, sesiones de grupo y encuestas (Creswell, 1994). Los estudios exploratorios son fuente de descubrimientos respecto de las variables que intervienen o participan en situaciones de consumo (Hernández, Fernández y Baptista, 1996). Complementariamente, los estudios descriptivos permiten caracterizar el fenómeno de consumo de acuerdo con las variables identificadas previamente (Hair, Bush y Ortinau, 2003).

A continuación, se describe con mayor detalle la muestra, los instrumentos y el procedimiento del estudio teniendo en cuenta que se desarrolló en dos fases: la primera fase se basó en la revisión de la literatura, lo que permitió generar el diseño de los instrumentos y su implementación tanto en entrevistas como en las sesiones de grupo, respectivamente; una segunda fase se centró en la realización de la encuesta en directo aplicada a 230 personas. Esta se fundamentó en la información recopilada en la fase inicial. Según los resultados conseguidos, se intentó dar una aproximación a una posible triangulación basada en la investigación cualitativa 
y cuantitativa de este estudio (Rodríguez Ruiz, 2005).

El proceso de investigación inicia con la búsqueda y revisión de artículos indexados en repositorios, todos relacionados con la construcción de marca en la industria musical y en los aplicativos para dispositivos móviles, como tabletas y teléfonos inteligentes.

Posteriormente, se contactaron fuentes primarias para la validación del instrumento y aplicación del trabajo de campo: un productor musical y uno radial. Se utilizaron otras fuentes secundarias (artículos de revistas, libros de texto, bibliografía) relacionadas con el problema de investigación propuesto, tras lo cual se logró identificar personas que tuviesen relación directa con el tema, como audiencias, artistas musicales, expertos de mercadeo digital y productores radiales. Se prosiguió a entrevistar a las personas que tienen un contacto directo con la industria y el mercado musical para que fuesen participes en el proceso investigativo en su papel de expertos (Taylor y Kinnear, 1998). En cuanto a audiencias, para las sesiones de grupo se contactaron jóvenes de edades entre 18 y 25 años, debido a que son un mercado cuyo consumo se ha inclinado hacia los medios digitales (Rodríguez, 2012).
La combinación de técnicas de entrevista, sesión de grupo y encuestas para la recolección de datos permitió mejorar la comprensión de las variables de análisis, lo que aportó validez y confiabilidad tanto en el desarrollo como en los resultados obtenidos.

Para cumplir los objetivos planteados, el diseño de este estudio contempló una fase de investigación cualitativa, basándose en criterios de selección no probabilístico, como el muestreo por expertos (Bonilla-Castro y Sehk, 2005; Goetz y LeCompte, 1988), y cuantitativa. Esto permitió explorar, descubrir e identificar los aspectos asociados a la creación de marca del artista musical. A continuación, se describe la fase 1 .

\section{Fase 1. Investigación exploratoria}

Para entender el comportamiento del consumidor actual hacia la comercialización, compra y venta de música, se realizó una indagación de carácter exploratorio haciendo uso de técnicas cualitativas. Dicha indagación estuvo orientada a recolectar y analizar las percepciones de grupos e interés. Se contó con la participación de especialistas (tres productores radiales), artistas (cinco músicos) 
y expertos digitales (cinco profesionales en mercadeo digital). El abordaje fue por medio de entrevistas en profundidad. Por otro lado, se indagó sobre los nuevos desarrollos tecnológicos, hábitos de compra y consumo de archivos digitales musicales. Para este propósito, se desarrollaron tres sesiones de grupo compuestas por un máximo de ocho participantes cuyas edades oscilaban entre 18-25 años.

El instrumento consistió en una guía de entrevista y guía de sesión de grupo. Fue construido y validado a partir de la revisión de la literatura y la consulta a dos expertos en el ámbito musical e investigativo. Después de las entrevistas, se realizaron tres sesiones de grupo dirigidas a jóvenes, cuyas ideas y hábitos de consumo correspondían a los intereses del estudio.

\section{Población}

En sintonía con el objetivo de investigación y el diseño propuesto, la población indagada estuvo dividida de acuerdo con los momentos de aplicación de las técnicas cualitativas de recolección de información. En este sentido, a continuación se describe cada uno de los subgrupos participantes.
1. Especialistas. Dentro de este subgrupo se contemplan profesionales relacionados con la industria musical y los medios de comunicación, como lo son tres productores radiales que fueron escogidos a conveniencia.

2. Artistas. Teniendo en cuenta que un artista en la música es una persona con habilidades y aptitudes musicales, los artistas entrevistados fueron escogidos a conveniencia de acuerdo con el reconocimiento, trayectoria y posibilidad de contacto. Este grupo estuvo constituido por cuatro artistas.

3. Expertos digitales. Considerando la importancia que tiene el mercadeo digital dentro de la industria musical actual, fueron seleccionados a conveniencia cinco gerentes de mercadeo digital.

Se realizaron tres sesiones de grupo para analizar las creencias, los sentimientos y los comportamientos hacia el consumo con dispositivos móviles y preferencia de artistas musicales que han incursionado en la era digital. La población seleccionada fueron jóvenes estudiantes universitarios con edades entre los $18 \mathrm{y}$ 
25 años pertenecientes a diferentes carreras relacionadas con mercadeo, comunicación, medios, usuarios en su mayoría de las TIC y de gustos musicales diversos. Dentro de las características de la población investigada se encontraron perfiles diversos con preferencias hacia géneros musicales como rock, punk, pop, salsa, merengue, electrónica y reggaeton, principalmente. En un porcentaje superior a $90 \%$ los asistentes manifestaron tener y hacer uso de las TIC con sus diferentes aplicativos con sus dispositivos móviles.

\section{Fase 2. Investigación descriptiva}

Se elaboró un cuestionario en directo de 10 preguntas que abordaban aspectos de edad, frecuencia de uso de aplicativos, aplicativos de mayor uso, frecuencia de descarga de aplicativos, disposición de pago por una aplicación, motivación para descargar aplicativos, utilización de aplicativo para relacionamiento con el artista, principales aspectos motivacionales que estimulen el uso de un aplicativo, disposición para pagos con un teléfono celular y frecuencia de compra de un aplicativo móvil. De 230 encuestas, 183 fueron efectivas, por lo que el margen de error fue de $7.7 \%$.

\section{Resultados}

Para el análisis de resultados, y según el objetivo del presente artículo, se empleó un método de análisis cualitativo de textos para representar las asociaciones entre los hallazgos a través de las sesiones de grupo y entrevistas. En este sentido, el primer análisis consistió en transcribir las entrevistas y las sesiones y después categorizar las verbalizaciones en unidades hermenéuticas ${ }^{1}$ creadas por los autores de acuerdo con la revisión de la literatura y la lógica interpretativa.

Es importante señalar que dada la extensión prevista para este documento no se expone el contenido de las diferentes unidades hermenéuticas construidas. A continuación, se hace referencia al análisis mediante mapas construidos en el software de análisis de información cualitativa Atlas.ti, versión 5.6, a partir de la integración hermenéutica y sistemática de verbalizaciones contenidas en unidades hermenéuticas. 


\begin{tabular}{|l|r|r|r|r|r|}
\hline \multicolumn{1}{|c|}{$\begin{array}{c}\text { Unidad } \\
\text { Hermenéutica }\end{array}$} & \multicolumn{1}{|c|}{$\begin{array}{c}\text { Artistas } \\
\text { Musicales }\end{array}$} & \multicolumn{1}{c|}{$\begin{array}{c}\text { (Productores } \\
\text { Radiales) }\end{array}$} & $\begin{array}{c}\text { Especialistas } \\
\text { En Marketing } \\
\text { Digital }\end{array}$ & $\begin{array}{l}\text { Focus } \\
\text { Group }\end{array}$ & Totales \\
\hline $\begin{array}{l}\text { Construcción del } \\
\text { artista }\end{array}$ & 67 & 28 & 8 & 42 & 145 \\
\hline $\begin{array}{l}\text { Tendencias de } \\
\text { consumo }\end{array}$ & 42 & 22 & 6 & 71 & 141 \\
\hline Branding digital & 15 & 7 & 39 & 27 & 88 \\
\hline Percepción de marca & 53 & 20 & 13 & 48 & 134 \\
\hline $\begin{array}{l}\text { Percepcín de } \\
\text { mercado }\end{array}$ & 52 & 19 & 18 & 19 & 108 \\
\hline Totales & 229 & 96 & 84 & 207 & 616 \\
\hline
\end{tabular}

\section{Análisis cualitativo:}

Figura 1: Representación de unidades hermenéuticas de entrevistas a productores radiales
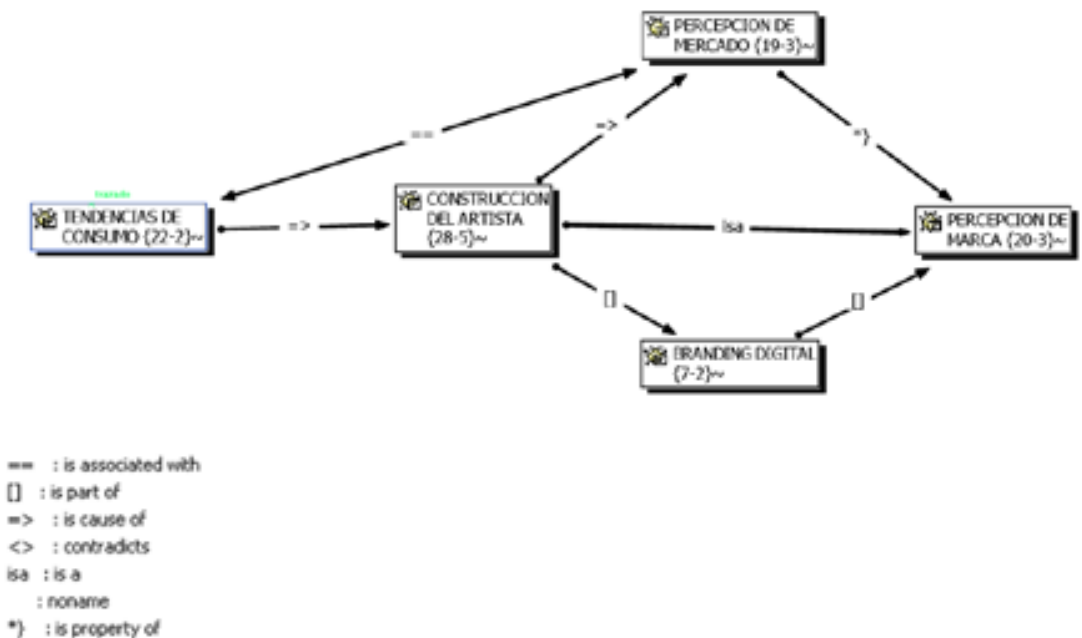

Se observa en la figura 1 que la productores radiales, e igualmente unidad hermenéutica mas represen- su propósito es desarrollar tanto una tativa es la construcción del artista percepción en el mercado (19 narraticon 28 narrativas. Dicha unidad es vas) y una percepción de marca (20). causa de las tendencias de consumo En complemento también se obserde acuerdo a la percepción de los va que el branding digital (7) es una 
alternativa o hace parte de los medios de construcción del artista:

"cuando tú te puedes caricaturizar o sea cuando tu eres caricaturizable es porque tienes un trabajo de marca en potencia muy bueno"

Se observa también que existe una relación para los productores radiales entre la percepción del mercado y las tendencias de consumo, es decir, el consumo de la industria musical ha variado de forma consistente de acuerdo a la evolución de las percepciones del mercado:

"los sellos disqueros no tienen billete ni le quieren meter, ya quieren vivir es de que el artista les haga la plata, ya no hacen artistas"

"tu compras una canción por 0,990 la puedas bajar gratis, es diferente a comprar un disco que te valía 70 u 80 mil pesos tocaba mandarlo a traer, ahora compras por US\$0,99 y te lo difieren con la tarjeta, eso cambia un poco el negocio de la música"

Finalmente la percepción de marca que tiene un artista desde el punto de vista de los productores radiales se encuentra constituida principalmente por los esfuerzos y/o construcción del mismo artista, las percepciones del mercado y las actividades de branding digital (que hacen también parte de la construcción del artista):

"el artista que se mira hacia dentro, el artista que no trata de imitar, el artista que está constantemente creando y en particular el artista humilde, es el que logra brillar, es aquel que rompe" 
Figura 2. Representación de unidades hermenéuticas de entrevistas a músicos

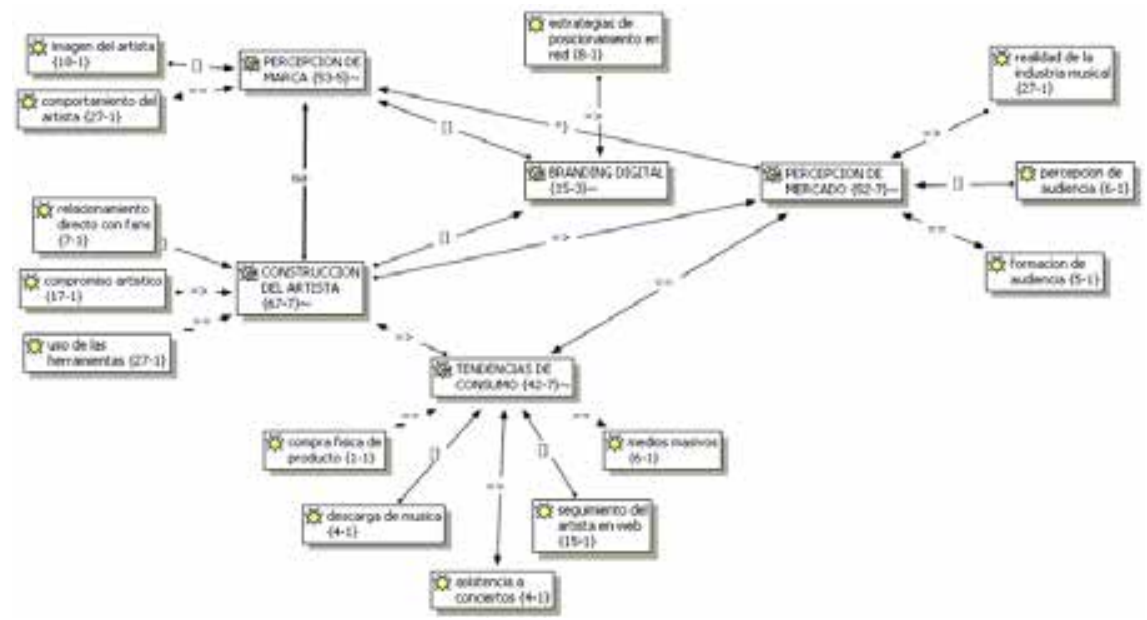

Respecto a la construcción de marca en el artista musical de acuerdo a las percepciones de músicos, se observa en la figura 2 que la unidad hermenéutica más representativa en el discurso corresponde a construcción del artista con 65 narrativas asociadas. Se observa en la figura 2 que las principales categorías que componen esta unidad son: el relacionamiento directo con fans (6), el compromiso artístico (17) y el uso de las herramientas orientadas a facilitar la construcción (27). Pro de ello lo constituyen verbalizaciones como:

"Ser artista es trabajar y trabajar, no autoproclamarse un artista. Es la gente quien le da ese titulo al artista"
"El artista de moda es mas rápido, pero siento que es más efímero. El de carrera tiene que trabajarse más, es de más tiempo, es de más dedicación pero al final de cuentas el resultado es mas grande".

Se observa también que la segunda unidad hermenéutica mas representativa fue la percepción de marca con 53 narrativas asociadas, siendo constituida principalmente por la imagen del artista y el comportamiento del mismo. En este caso es importante describir que el comportamiento del artista se encuentra asociado a la precepción de marca mientras que la imagen es parte; dicho fenómeno se puede ilustrar mediante verbalizaciones como: 
"La música se tiene también que ver y cuando uno oye la canción uno la ve... así no la estés viendo pero estas imaginando y uno tiene una imagen de esa canción"

"El músico que no tiene clara su raíz esta perdido, esta equivocado".

Respecto a la unidad hermenéutica de percepción de mercado constituida por 52 narrativas se observa que la realidad de la industria musical (27) hace parte de la misma; la percepción de la audiencia (6) hace parte de la percepción del mercado y la formación de audiencia se encuentra asociada (5). Es de aclarar que las categorías de percepción y formación de audiencia han sido vinculadas a la percepción del mercado de manera directa y no a la unidad de percepción de marca, debido a que la naturaleza de las verbalizaciones hablan del mercado y no de la marca, como por ejemplo:

"Somos marcarios en Colombia. somos muy dados a rendir tributo a eso. Por consiguiente también nos encantan las marcas. No creo que lo hayan aprovechado muy bien los artistas"
"Si hago parte del entretenimiento, hago entretenimiento y hago show business pues me termino convirtiendo en una marca"

La unidad hermenéutica de tendencias del consumo con 42 narrativas se encuentra constituida por las descargas de música (4) y el seguimiento del artista en la web (15) los cuales hacen parte de este fenómeno. Igualmente se observa que la compra física del producto (1), la asistencia a conciertos (4) y el uso de medios masivos (6) se encuentran asociadas a dichas tendencias. Como referente:

"Genial es poder escuchar una canción, meterse a un aplicativo móvil, buscar cual es la canción, en Shazam, en Sounhoud, en Youtube, tener la letra y de una vez que me conecta a Itunes, pagar 0,99 centavos es muy poco".

Finalmente al analizar la unidad hermenéutica de branding digital con 15 narrativas se observa que la categoría de estrategias de posicionamiento en red (8) es una causa de dicha actividad. Igualmente la construcción del artista hace parte del branding digital. "yo pienso que hoy en día un artista tiene que estar de socio con un nerd al 
lado (...) no puede ser solo el artista y su piano, tiene que ser el artista, el piano, y el nerd al lado, un man que lo va a filmar, que le va a dar su imagen, que lo va a mover en las redes, va a estar 4 o 5 horas en las redes al día moviendo las actualidades del artista porque si no no sale, hay mucha competencia hoy".

Figura 3. Representación de unidades hermenéuticas de entrevistas a especialistas en marketing digital

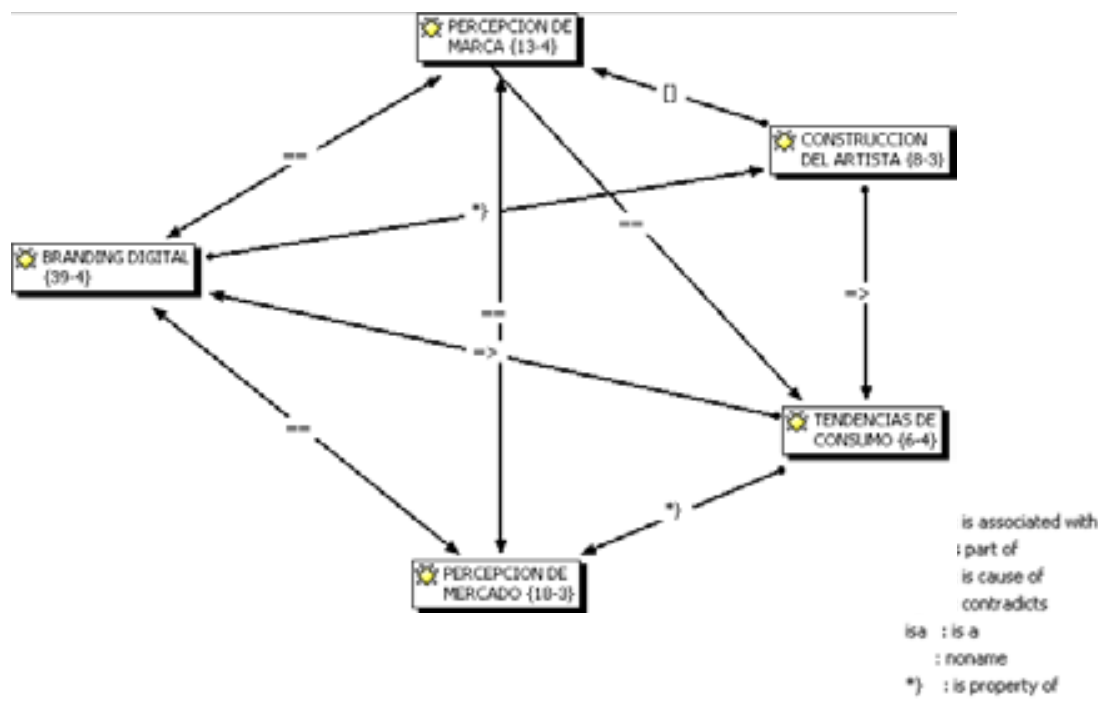

Se observa en la figura 3 que la unidad hermenéutica mas representativa es la denominada branding digital con 39 narrativas. Dicha unidad es asociada con la percepción de marca (13) y percepción de mercado (18) que tienen los expertos digitales sobre el tema. La construcción del artista (8) que es propiedad del branding digital, a pesar de contar con menos narrativas también es parte de la percepción de marca (8) y es causa de las tendencias de consumo (6), la cual a su vez es propiedad de la percepción de mercado sobre los cambios en la industria musical y los tipos de audiencias. Un ejemplo de esto son las siguientes verbalizaciones:

"Hay que hacer es un gran trabajo... yo cómo hago para que la gente se entere que existe una aplicación, que la encuentren y la descarguen, ese es el gran reto realmente" 
"Hay una serie de consideraciones bien interesantes para tener... hay que ser muy inteligente para no generar percepciones negativas, para ser certero."
"Se debe entender que cada vez mas personas están conectadas en internet... dependiendo de lo que es mi empresa, mi producto, mi marca, yo puedo generar valor... que pueda tener un impacto importante."

Figura 4. Representación de unidades hermenéuticas desde las sesiones de grupo

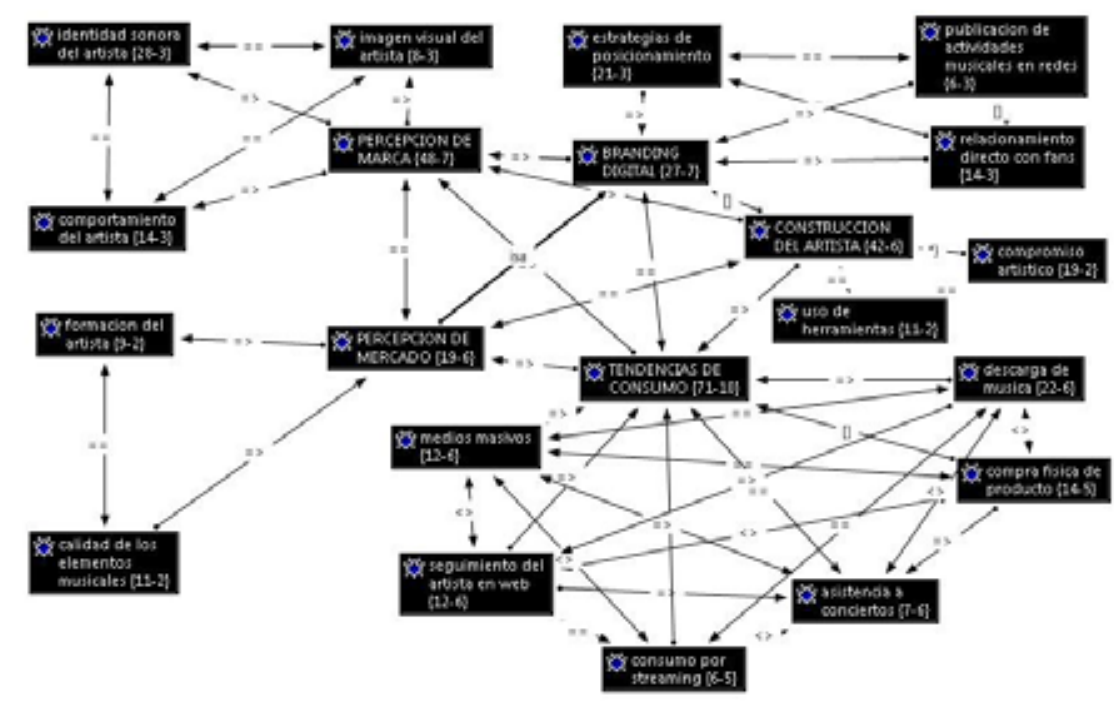

Debido a las restricciones de extensión del documento, el análisis de las tres sesiones de grupo se presenta en un solo mapa de relaciones de contenido cualitativo categorizado. Se observa en la figura 4 , que respecto a la construcción de marca, desde el punto de vista del mercado, se puede identificar que la unidad hermenéutica más representativa es la de tendencias de consumo con 71 narrativas asociadas. Se observa en la figura anterior, que las principales categorías que componen esta unidad son la percepción de marca (48), construcción del artista (42), Percepción de mercado (19), Branding Digital (27). Opiniones como las siguientes confirman una relación directa:

"lo que mas potencializa a un artista creo que es lo visual, que es con 
lo que más impacta, también sus sonidos y la forma en que se dirige al público a través de los medios".

Para conseguir una construcción del artista, se debe tener en cuenta la base de las tendencias de consumo actuales, como la descarga de música (22), seguimiento del artista en web (12) y el uso constante de los medios masivos (12); puesto que de ahí parte las estrategias de comunicación que le permitan al artista conseguir los objetivos planteados, así lo ratifica el siguiente verbatim:

“ya no compro $C D$ 's porque encuentro la música gratis en internet, entonces porque voy a pagar por algo que esta en la red".

De igual forma se hace primordial aplicar la unidad hermenéutica estrategias de Branding Digital, que conlleven a un constante relacionamiento con los fans (14) y a mantener al público informado a través de las redes sociales(6), puesto que ello podría genera afecto y respeto por parte de los seguidores o fans, además mantener siempre una identidad musical clara (28) y un comportamiento adecuado (14) a la imagen visual (8), que se desee promocionar por parte del artista.
Finalmente, el análisis de la población de millenials estuvo constituido por los asistentes a las sesiones de grupo, quienes permitieron identificar que el mercado se enfoca mucho más por la percepción que se tenga del artista y los cambios que se han presentado en cuanto al consumo y sociabilización de contenidos relacionados con marcas, que despiertan el interés de sus grupos de nicho que se comunican a través de redes sociales.

\section{Análisis descriptivo}

Para complementar los resultados de la fase cualitativa se realizó un trabajo de campo de naturaleza cuantitativa basado en 230 encuestas, de las cuales al ser depuradas se obtuvieron 183 casos efectivos. Entre los resultados descriptivos de la muestra se puede destacar que $42,4 \%$ de los encuestados están entre 26 y 35 años, 30,9\% entre 18 y 25 años mientras que el $26,6 \%$ restante pertenece a los menores de 18 años.

En cuanto a los encuestados con edades entre 18 y 25 años, un $51,8 \%$ manifestó que el dispositivo móvil que mas utiliza es smartphones, seguido de un $24,5 \%$ que utiliza teléfono móvil tradicional. Respecto a los tipos de aplicativos móviles mas utilizados predominan los de 
comunicación con $58,3 \%$ seguidos por los de entretenimiento con un $43,9 \%$. Un 66,2\% manifestó si estar interesado en utilizar un aplicativo móvil para estar en contacto con su artista preferido; los principales motivadores para este uso son los videos con un $63,3 \%$ seguido de la opción de comunicarse directamente con el artista con un $28,8 \%$.

Los dispositivos mas utilizados para el consumo de música son el teléfono móvil con el $64,7 \%$, seguido por el computador con $39,6 \%$. Se identifica que en la percepción que tiene el público con respecto a cómo un artista logra obtener mas seguidores, se destacan la innovación que este tenga en sus producciones musicales y audiovisuales con un $42,4 \%$, el género musical que interprete con $41 \%$ y la percepción de marca que haya generado en el público con un $23 \%$.

Un 33,1\% de los encuestados manifestó estar atento a las novedades de su artista predilecto de forma semanal, mientras que un 25,9\% lo hace mensualmente. Se reflejó en la muestra un bajo interés en cuanto a la recepción de información del artista por parte de los fans, sin embargo los medios que en algunos casos mas llamaron la atención para dicho propósito fueron los aplicativos móviles con $39,6 \%$, redes sociales con 30,2 \% y correo electrónico con $27,3 \%$ respectivamente.

El segmento de la muestra con edades entre 26 y 35 años manifestó que el dispositivo móvil que mas utiliza es el sistema de navegación en automóvil con un $34,9 \%$ seguido de un $27,9 \%$ en sistemas de televisión e internet y un 25,6 \% en smartphones. Respecto a los tipos de aplicativos móviles mas utilizados predominan los de información con $62,8 \%$ seguido por servicios de localización con un $27,9 \%$. Un $93 \%$ manifestó si estar interesado en utilizar un aplicativo móvil para tener un contacto con su artista preferido; el único motivo manifestado por este grupo para dicho contacto es recibir información del artista.

Los dispositivos mas utilizados para el consumo de música son el computador con el $41,9 \%$ y la radio con el $34,9 \%$. Se identifica que en la percepción del público con respecto a cómo un artista logra obtener mas seguidores, se destacan la innovación que este tenga en sus producciones musicales y audiovisuales con un 51,2\%, la percepción de marca que haya generado en el público con un $27,9 \%$ y el género musical que interprete con $23,3 \%$. Un $60,5 \%$ de los 
encuestados manifestó estar atento a las novedades de su artista predilecto de forma semanal, mientras que un $20,9 \%$ lo hace quincenalmente. Un $90,7 \%$ manifestó no estar interesado en recibir notificaciones de su artista por medio de un aplicativo móvil; el $74,4 \%$ prefiere que sea por redes sociales y un $18,6 \%$ por mensajes de texto.

La prueba de Kolmogorov Smirnov aplicada a cada una de las variables de la encuesta indica que las variables cumplen con los criterios de normalidad. Al hacer pruebas de asociación entre variables como: edad, dispositivos móviles mas utilizados, percepción de construcción de marca, preferencias para el consumo musical y motivaciones para el consumo de aplicativos móviles, se halló que el grupo mas propenso al consumo de marcas musicales es el menor de 18 años, siendo también el mas consistente para la recepción de una construcción de marca a través de aplicativos móviles. Por tanto se infiere este como un nicho de mercado altamente relacionado con los avances tecnológicos que consume principalmente aplicaciones móviles para fines de entretenimiento y se conecta con mayor frecuencia a través de smartphones.
Al realizar un ejercicio de clasificación de la población se usó un cluster bietápico para revisar la existencia de grupos con distintos comportamientos, lo cual se pudo evidenciar durante el análisis. La medida de silueta, cohesión y separación de ese análisis resulta suficiente para afirmar que existen 2 agrupaciones cuyas características son:

El grupo 1: se encuentran entre los 18 y 25 años, hacen uso de Smartphones, su consumo de aplicaciones móviles se usa en gran porcentaje para comunicación, están dispuestos a hacer uso de un aplicativo móvil que los conecte con sus artistas favoritos, para lo cual su principal motivación son los videos de la banda musical y usualmente revisan información propia de sus artistas a través de internet.

El grupo 2: rango de edad entre los 26 y 35 años, hacen uso de internet y TV, los aplicativos que más utilizan son los que les permiten mantenerlos informados, estarían dispuestos a adquirir una aplicación móvil que les permita estar conectados con su artista favorito, es por ello que su principal motivo para hacer uso de un aplicativo relacionado con su artista es para obtener información detallada de las actividades; además consideran que la 
innovación en el artista es el camino más idóneo para cautivar a un mercado más amplio. Este grupo estaría dispuesto a recibir información de su artista a través de redes sociales.

Si bien se demostró que hay 2 grupos: los de 18 a 25 años son un grupo que si cree en la marca, utiliza los dispositivos, están atentos a los avances tecnológicos, además se apegan menos con los objetos materiales relacionados con música. El segmento de 26 a 35 años a pesar de consumir habitualmente productos musicales y tener mayor facilidad para la compra de productos por vía digital, no se reflejó tan firme en el seguimiento de un artista por medio de aplicativos móviles como los de menor edad.

\section{Discusión}

En Colombia, la oferta artística musical ha mostrado un incremento durante los últimos años. Eventos como el Bogotá Music Market (Bomm) organizado por la Cámara de Comercio de Bogotá y que en 2014 realiza su tercera edición, mostró en 2013 el interés de un mercado poco atendido, el musical, con la inscripción de 1300 proyectos y la participación 665 artistas para relacionar sus obras con marcas comerciales en busca de la generación de posteriores negocios (300 líderes, 2013).

Por otro lado, los dispositivos móviles han tenido un alto crecimiento durante los últimos años. La penetración de telefonía celular en Colombia ha llegado al $100 \%$ y de smatphones al $30 \%$ en el 2012. Adicionalmente, aspectos como la inversión realizada a la publicidad móvil alcanzó los 1.774 millones de pesos durante el primer semestre de $2013 \mathrm{y}$ un $61 \%$ de usuarios acceden a internet a través de sus smartphones, índice que ratifica una fuerte tendencia al consumo de contenidos digitales a través de dispositivos móviles por parte de la generación denominada los millenials (P\&M, 2013). De esta manera la comunicación a través de aplicativos móviles es una forma de potencializar el mercadeo para un artista musical, favoreciendo la construcción de su marca con la utilización del mix de mercadeo que va de las 4 a las 8 p's, las cuales son las mismas tanto para la promoción offline como online, con el componente que permite al campo digital segmentar y medir con exactitud en tiempo real al público objetivo, generando un mensaje personalizado y no intrusivo, distante de la forma en que se dirige una promoción por los medios masivos 
tradicionales (Pousttchi \& Wiedemann, 2006).

La presente investigación ha permitido identificar los principales aspectos que contribuyen a la construcción de marca de un artista sobre el público, avalando el desarrollo tecnológico y aporte que un aplicativo móvil puede brindar a un músico para fortalecer su marca. Los resultados obtenidos en la etapa cualitativa $y$ en la cuantitativa se triangularon para construir la discusión y conclusiones. Cabe resaltar que dentro del consumo creciente de contenidos digitales en smartphones, la innovación y el desarrollo a nivel de software y hardware pueden ser factores fundamentales para la construcción del artista y su relacionamiento con el mercado, por medio de la transformación de un dispositivo para fines muy concretos que favorezcan al artista (Oh, J. Et al, 2010).

Según los resultados de la fase cualitativa (sesiones de grupo y entrevistas a artistas), elementos como la propuesta lirica, composición, sonoridad (musicalidad), propuesta visual y puesta en escena inciden directamente en la construcción de marca en el artista. La conexión entre el artista con su propuesta musical y su público es fundamental, puesto que le permitirá obtener información para generar bases sólidas para la consolidación de su marca, siendo influyente en la preferencia por parte del público, específicamente el de los millenials.

De acuerdo con los resultados cuantitativos, se contempla a los dispositivos móviles con apoyo de las aplicaciones, como herramientas idóneas para alcanzar un alto grado de relacionamiento y en consecuencia fortalecer la marca artística teniendo siempre presente los comentarios de sus seguidores; de igual forma es preciso el compromiso que el artista tenga con su obra y con sus fans, comportándose consecuentemente con los valores de marca proyectados durante su carrera o en dado caso transformándolos estratégicamente para lograr impactar y obtener un alto grado de aceptabilidad; permanecer atento a la comunicación con los fans por medio de las TICs es un aspecto del cual ningún artista debe prescindir.

Aunque los artistas tienen conocimiento con respecto a la masificación de dispositivos móviles y las aplicaciones que les permitirían ayudar de forma efectiva la construcción de su marca, se pudo percibir que no existe un profundo conocimiento sobre el potencial que tienen 
estas herramientas para beneficio del artista, lo cual se ve reflejado en las muchas propuestas musicales colombianas existentes en la actualidad y la pobre presencia de las mismas en los medios digitales de vanguardia. Es posible corroborar el cambio inminente que ha tenido la industria musical durante los últimos años, aumentando la desmaterialización del bien físico, generando nichos de mercado que valoran y adquieren productos físicos - como un vinilo o un $\mathrm{CD}$ - por razones alternas al consumo masivo (bien de lujo, coleccionista o regalo) y haciendo del producto musical un conjunto de datos descargable, de fácil almacenamiento y transporte.

La era digital está cambiando el comportamiento del consumidor, lo que ha llevado a muchas marcas encontrar en las diversas actividades diarias de los usuarios, la forma de promocionarse y estar en contacto directo con ese mercado real a través de aplicativos móviles. Esto ha facilitado la comercialización de forma inmediata de productos y servicios a través de estos nuevos mecanismos que están al alcance de millones de usuarios digitales. La industria musical ha volcado parte de sus estrategias de promoción de artistas hacia los medios digitales, sin embargo es importante ofrecer innovación y funcionalidad a dichas herramientas para que pueda ser masificado y a la vez retroalimentado por los mismos usuarios, quienes constantemente están aportando y enriqueciendo con información, que puede favorecer en la percepción sobre un artista determinado. Si un artista quiere construir su marca, debe tener presente las nuevas tendencias de consumo del mercado y las motivaciones del mismo, valiéndose de las estrategias y tácticas del mercadeo en pro del branding digital y de una correcta utilización de los recursos financieros al momento de invertir en una campaña de mercadeo (Edelman, D. 2010).

Es importante tener presente que el desarrollo tecnológico va a pasos agigantados y que la tendencia de consumo esta ligada al desarrollo de nuevas tecnologías. La industria musical se encuentra en constante replanteamiento de sus estrategias de venta, las cuales se inclinan hacia el marketing digital. Es probable que en un par de años los productores y managers hayan cambiado su forma de promocionar sus artistas, por tal razón debe hacerse seguimiento a las tendencias de consumo del mercado y al grado de profesionalización que el artista desarrolle en cuanto a la 
construcción de su marca por medio del branding digital. Se recomienda identificar en futuras investigaciones el efecto que genera en el público la constante comunicación y promoción de los artistas a través de los aplicativos móviles, realizar un análisis sobre la efectividad que puedan alcanzar los mensajes enviados a través de aplicaciones en dispositivos móviles y su aceptación por parte del mercado.

\section{Referencias bibliográficas}

300líderes (2013, 23 de octubre). BOmm - Bogota music market 2013. Recuperado de http://300-lideres. blogspot.com/2013/10/bomm-bogota-music-market-2013.html

Aaker, D. y Joachimsthaler, E. (2005). Liderazgo de marca. España: Deusto.

AdWords. Anuncie su empresa en Google. Recuperado de https://accounts.google.com/ServiceLogin?s ervice $=$ adwords $\&$ continue $=$ https: $/ /$ adwords.google.com/um/gaiaauth? apt\%3DNone\%26ltmpl\%3Djfk\&hl $=$ es_ES\&ltmpl $=$ jfk \&passive $=86400$ $\&$ skipvpage $=$ true $\&$ sacu $=1 \&$ sarp $=1$

Aliado Digital. Consumo musical: el dominio de YouTube sobre MySpace.
Recuperado de http://www.aliadodigital.com/2010/05/consumo-musicalyoutube-es-mas-importante-quemyspace/

American Marketing Association (2013). Definition of marketing. Recuperado de https://www.ama.org/ AboutAMA/Pages/Definition-ofMarketing.aspx

Flores, J. I. A., Pérez, M. J. S. y Blanco, V.F. (2010). El marketing de la cultura y las artes: una evolución. Revista $\mathrm{Na}$ cional de Administración, 1(1), 23-36. Báez, J. y Tudela, P. de (2007). Investigación cualitativa. ESIC.

Barrere, R. (2012, marzo). La investigación y el desarrollo en TIC en Iberoamérica. Situación actual y tendencias. Papeles del Observatorio, 5. Recuperado de http://www.observatoriocts.org/files/Archivo\%20Documental/Papeles\%20del\%20Observatorio/InvestigacionyDesarrollo_EDITADO_FINAL_DEFINITIVO.pdf

Bonilla-Castro, E. y Sehk, P. R. (2005). Más allá del dilema de los métodos: la investigación en ciencias sociales. Norma. 
Brookshire, R. (2007). The iPod revolution: coming to a classroom near you. En Organizational Systems Research Association Conference (pp. 1-7).

Boorsma, M. (2006). A strategic logic for arts marketing. International Journal of Cultural Policy, 12, 73-92.

Carrero, J. S. y Pulido, P. C. (2012). De cara al prosumidor: producción y consumo empoderando a la ciudadanía 3.0. Revista ICONO14. Revista científica de Comunicación y Tecnologías emergentes, 10(3), 62-84.

Cereijo, I. (2014, 9 de enero). Música, social media y tendencias digitales para 2014. Dosdoce.com. Recuperado de http://www.dosdoce.com/articulo/ opinion/3887/musica-social-mediay-tendencias-digitales-para-2014/

Colombia, Ministerio de Tecnologías de la Información y las Comunicaciones (2010). Percepción, usos y hábitos frente a las tecnologías de la información y la comunicación. Recuperado de http://www.vivedigital. gov.co/foros/comentarios/encuesta percepcion_TIC.pdf

Creswell, J. W. (2006). Diseño de investigación. Aproximaciones cualitativas y cuantitativas. Universidad de
Buenos Aires. Facultad de Sociología. Sage Capitulo, 9, 143-171.

Ding, C. G. y Lin, C. H. (2012). How does background music tempo work for online shopping? Electronic Commerce Research and Applications, 11(3), 299-307.

Edelman, D. (2010). The digital age: you're spending your money in all the wrong places. Harvard Business Review. Recuperado de https://hbr. org/2010/12/branding-in-the-digitalage-youre-spending-your-money-inall-the-wrong-places

Gobé, M. (2005). Branding emocional: el nuevo paradigma para conectar las marcas emocionalmente con las personas.

Goetz, J. P. y LeCompte, M. D. (1988). Etnografía y diseño cualitativo en investigación educativa. Madrid: Morata. Goldsmith, R. E. (1999). The personalised marketplace: beyond the 4Ps. Marketing Intelligence \& Planning, 17(4), 178-185.

Hair, B. y David, J. Ortinau (2003). Investigación de mercados en un ambiente de información cambiante. México: McGraw-Hill. 
Hipertextual. Google introduce cambios en Ads para ayudar a hacer decisiones financieras. Recuperado de http://hipertextual.com/archivo/2012/04/google-introduce-cambios-en-ads-para-ayudar-a-hacer-decisiones-financieras/

Hipertextual. Historia de la publicidad: la evolución del marketing digital. Recuperado de http://hipertextual.com/archivo/2011/12/historiapublicidad/

Hirschman, E. C. (1983). Aesthetics, ideologies and the limits of the marketing concept. The Journal of Marketing, 45-55.

Hoyos, N. (2008). Marketing: más de 100 años de historia. Génesis y evolución de un concepto. Lecturas Seleccionadas de Asomercadeo.

Kazadi, M. (2010). The role of musical instruments in the globalization of music. Revista Comunicar, 34, 83-89.

Kotler, P. (1973). Atmospherics as a marketing tool. Journal of Retailing, 49(4), 48-64.

Kotler, P. (1993). Dirección de la mercadotécnica. análisis, planeación, implementación y control (7. ${ }^{\mathrm{a}}$ ed.). México: Prentice Hall.

Kotler, P., Armstrong, G., Ayala, L. E. P. y Félix, M. B. (2007). Marketing: versión para Latinoamérica. México: Pearson Prentice Hall.

Liberos, E. (2013). El libro del marketing interactivo y la publicidad digital. ESIC.

López, J. (2005). Manual urgente para radialistas apasionados. Ediciones Paulinas.

Mantecón, A. R. (2010). Del público al prosumidor. Nuevos retos para los estudios de consumo cultural. Entretextos, 6, 37-42.

Mukuma, K. (2010). The role of musical instruments in the globalization of music. Revista Comunicar, 17(34), 83-88.

Ng, E. S., Schweitzer, L. y Lyons, S. T. (2010). New generation, great expectations: a field study of the millennial generation. Journal of Business and Psychology, 25(2), 281-292.

Ogden, J. R., Ogden, D. T. y Long, K. (2011). Music marketing: a history and landscape. Journal of Retailing 
and Consumer Services, 18(2), 120125.

Oh, J., Herrera, J., Bryan, N. J., Dahl, L. y Wang, G. (2010, junio). Evolving the mobile phone orchestra. En Proceedings of the International Conference on New Interfaces for Musical Expression (pp. 82-87).

P\&M (2013, 12 de noviembre). COLOMBIA: penetración del $100 \%$ en telefonía celular y del $30 \%$ en smartphones. Recuperado de http:// www.revistapym.com.co/noticias/ marketing-movil/colombia-penetracion-100-telefonia-celular30-smartphones

Pousttchi, K. y Wiedemann, D. G. (2006, junio). A contribution to theory building for mobile marketing: categorizing mobile marketing campaigns through case study research. En Mobile Business, 2006. ICMB'06. International Conference on (pp. 1-1). IEEE.

Reyes, G. E. (2001). Teoría de la globalización: bases fundamentales. Nómadas: revista crítica de ciencias sociales y jurídicas, 3,8 .

Rodríguez, J. (2012). El consumidor digital Colombiano. Recuperado de http://es.slideshare.net/juancrodriguezgomez/el-consumidor-digitalcolombiano? related $=2$

Rodríguez Ruiz, O. (2005). La triangulación como estrategia de investigación en ciencias sociales. Revista de Investigación en Gestión de la Innovación y Tecnología, 31.

Schiffman, L. G. y Kanuk, L. L. (2010). Comportamiento del consumidor (10. ${ }^{\mathrm{a}}$ ed.). México: Pearson

Shankar, V. y Balasubramanian, S. (2009). Mobile marketing: a synthesis and prognosis. Journal of Interactive Marketing, 23(2), 118-129.

Silverman, S. N. (1995). An historical review and modern assessment of the marketing mix concept. En $7^{\mathrm{a}}$ Marketing History Conference Proceedings (vol. 11).

Smith, M. (2011). El nuevo marketing relacional. Anaya.

Solomon, M. R., Polegato, R. y Zaichkowsky, J. L. (2009). Consumer behavior: buying, having, and being (vol. 6). Upper Saddle River, NJ: Pearson Prentice Hall.

Taken Smith, K. (2012). Longitudinal study of digital marketing strategies 
targeting Millennials. Journal of Consumer Marketing, 29(2), 86-92.

Taulet, A. C. y Contrí, G. B. (2006). Influencias del posmodernismo en marketing y comportamiento del consumidor: ¿el fin de la era del marketing? Revista Española de Investigación de Marketing, 10(17), 7-26.

Taylor, J. R. y Kinnear, T. C. (1998). Investigación de mercados. Un enfoque aplicado (5. ${ }^{\mathrm{a}}$ ed.). México: Editorial McGraw-Hill.
Vaccaro, V. L. y Cohn, D. Y. (2004). The evolution of business models and marketing strategies in the music industry. International Journal on Media Management, 6(1-2), 46-58.

Van Waterschoot, W. y Van den Bulte, C. (1992). The 4P classification of the marketing mix revisited. The Journal of Marketing, 83-93. 